\title{
BACKWARD STOCHASTIC DIFFERENTIAL EQUATIONS WITH STOCHASTIC MONOTONE COEFFICIENTS
}

\author{
K. BAHLALI, A. ELOUAFLIN, AND M. N'ZI \\ Received 25 October 2003 and in revised form 24 June 2004
}

We prove an existence and uniqueness result for backward stochastic differential equations whose coefficients satisfy a stochastic monotonicity condition. In this setting, we deal with both constant and random terminal times. In the random case, the terminal time is allowed to take infinite values. But in a Markovian framework, that is coupled with a forward SDE, our result provides a probabilistic interpretation of solutions to nonlinear PDEs.

\section{Introduction}

Backward stochastic differential equations (BSDEs), introduced by Pardoux and Peng [10], have been intensively studied in the last years. This class of equations is a powerful tool to give probabilistic formulas for solutions of semilinear partial differential equations (PDEs). We refer the reader to $[8,9]$ for a good presentation of BSDEs and their connections to PDEs. These equations have found a broad area of applications, namely, in stochastic optimal control (see [7]), mathematical finance (see [6]). Many existence and uniqueness results have been proved in relaxing the uniform Lipschitz condition on the coefficient. Among others, we refer to those with monotonicity condition (see $[1,3,4])$. In this setting (in relaxing the Lipschitz condition), Bender and Kohlmann [2] recently considered the so-called stochastic Lipschitz condition introduced by El Karoui and Huang [5] and dealt with BSDEs with random terminal time. Indeed, the Lipschitz coefficient is allowed to be an $\mathscr{F}_{t}$-adapted process. Doing so, one must reinforce the integrability conditions on the data as well as on the solutions. The interest in this type of extension of the classical existence and uniqueness result comes from the fact that, in many applications, the usual Lipschitz condition cannot be satisfied. For example, the pricing of a European claim is equivalent to solving the linear BSDE

$$
\begin{gathered}
-d Y_{t}=[r(t) Y(t)+\theta(t) Z(t)] d t-Z(t) d W_{t}, \\
Y_{T}=\xi
\end{gathered}
$$

Copyright (C) 2004 Hindawi Publishing Corporation Journal of Applied Mathematics and Stochastic Analysis 2004:4 (2004) 317-335 2000 Mathematics Subject Classification: 60H10, 60H20

URL: http://dx.doi.org/10.1155/S1048953304310038 
where $\xi$ is the contingent claim, $r(t)$ is the interest rate, $\theta(t)$ is the risk premium vector, and $T$ is the terminal time. Both $r(t)$ and $\theta(t)$ are not bounded in general. Therefore, the generator satisfies the so-called stochastic Lipschitz condition which means that the Lipschitz constant is a stochastic process.

In this paper, we continue this study by considering BSDEs with stochastic monotone coefficients. For example, our result treats generators of the following type: $f(t, y, z)=$ $\mu(t) g(t, y)+h(t, 0, z),(t, y, z) \in[0, T] \times \mathbb{R} \times \mathbb{R}^{d}$, where $\mu(t)$ is a nonnegative $\mathscr{F}_{t}$-adapted process, $g$ satisfies a monotonicity condition in $y$ and $h$ is stochastic Lipschitzian in $z$. It is not possible to apply the results of $[2,4]$. Our aim is to prove the existence and uniqueness of solutions for both constant and random terminal times. When the terminal time is random, it is allowed to take values in $[0,+\infty]$.

The paper is organized as follows. In Section 2, we give some notations, state the assumptions, and define the BSDEs we are concerned with. Section 3 treats the nonrandom terminal time case, and Section 4 deals with the random one.

\section{Notations, assumptions, and definitions}

2.1. Notations. Let $W=\left\{W_{t}, \mathscr{F}_{t}, t \geq 0\right\}$ be an $n$-dimensional Brownian motion defined on a probability space $\left(\Omega, \mathscr{F}_{\mathscr{F}}, \mathbb{P}\right)$. $\left\{\mathscr{F}_{t}, t \geq 0\right\}$ stands for the natural filtration of $W$, augmented with the $\mathbb{P}$-nul sets of $\mathscr{F}$. The inner product of $\mathbb{R}^{d}$ is denoted by $\langle\cdot, \cdot\rangle$ and the Euclidean norm by $|\cdot|$. The norm of $\mathbb{R}^{d \times n}$ is denoted by $|Z|^{2}=\operatorname{tr}\left(Z Z^{*}\right)$.

Let $\beta>0, \tau$ be a positive real-valued random variable and $a$ a nonnegative $\mathscr{F}_{t}$-adapted process. We define the increasing process $A(t)=\int_{0}^{t} a^{2}(s) d s$ and consider the spaces:

$$
\begin{aligned}
& L^{2}\left(\beta, a, \tau, \mathbb{R}^{d}\right)=\left\{\begin{array}{l}
\xi ; \mathbb{R}^{d} \text {-valued, } \mathscr{F}_{\tau} \text {-measurable random variables } \\
\text { such that }\|\xi\|_{\beta}^{2}=\mathbb{E}\left(e^{\beta A(\tau)}|\xi|^{2}\right)<+\infty
\end{array}\right\}, \\
& L^{2}\left(\beta, a,[0, \tau], \mathbb{R}^{d}\right)=\left\{\begin{array}{l}
Y ; \mathbb{R}^{d} \text {-valued, } \mathscr{F}_{t} \text {-adapted processes such that } \\
\|Y\|_{\beta}^{2}=\mathbb{E}\left(\int_{0}^{\tau} e^{\beta A(s)}|Y(s)|^{2} d s\right)<+\infty
\end{array}\right\}, \\
& L^{2, a}\left(\beta, a,[0, \tau], \mathbb{R}^{d}\right)=\left\{\begin{array}{l}
Y ; \mathbb{R}^{d} \text {-valued, } \mathscr{F}_{t} \text {-adapted processes such that } \\
\|a Y\|_{\beta}^{2}=\mathbb{E}\left(\int_{0}^{\tau} e^{\beta A(s)} a^{2}(s)|Y(s)|^{2} d s\right)<+\infty
\end{array}\right\}, \\
& L^{2, c}\left(\beta, a,[0, \tau], \mathbb{R}^{d}\right)=\left\{\begin{array}{l}
Y ; \mathbb{R}^{d} \text {-valued, continuous } \mathscr{F}_{t} \text {-adapted processes } \\
\text { such that }\|Y\|_{\beta, c}^{2}=\mathbb{E}\left(\sup _{0 \leq s \leq \tau} e^{\beta A(s)}|Y(s)|^{2}\right)<+\infty
\end{array}\right\} .
\end{aligned}
$$

$L^{2}\left(\beta, a,[0, \tau], \mathbb{R}^{d}\right)$ is a Banach space with the norm $\|\cdot\|_{\beta}$. Consequently,

$$
M(\beta, a, \tau)=L^{2, a}\left(\beta, a,[0, \tau], \mathbb{R}^{d}\right) \times L^{2}\left(\beta, a,[0, \tau], \mathbb{R}^{d \times n}\right)
$$

is a Banach space with the norm $\|(Y, Z)\|_{\beta}^{2}=\|a Y\|_{\beta}^{2}+\|Z\|_{\beta}^{2}$. We denote by $\mathcal{M}^{c}(\beta, a, \tau)$ the subspace of $\mathcal{M}(\beta, a, \tau)$ defined as follows:

$$
M^{c}(\beta, a, \tau)=\left(L^{2, a}\left(\beta, a,[0, \tau], \mathbb{R}^{d}\right) \cap L^{2, c}\left(\beta, a,[0, \tau], \mathbb{R}^{d}\right)\right) \times L^{2}\left(\beta, a,[0, \tau], \mathbb{R}^{d \times n}\right) ;
$$

and consider the norm $\|(Y, Z)\|_{\beta, c}^{2}=\|Y\|_{\beta, c}^{2}+\|a Y\|_{\beta}^{2}+\|Z\|_{\beta}^{2}$ on $M^{c}(\beta, a, \tau)$. 
Remark 2.1. If $a$ and $b$ are two nonnegative $\mathscr{F}_{t}$-adapted processes such that $b>a$, then $L^{2}\left(\beta, b,[0, \tau], \mathbb{R}^{d}\right) \subset L^{2}\left(\beta, a,[0, \tau], \mathbb{R}^{d}\right)$. Consequently, $\mathcal{M}^{c}(\beta, b, \tau) \subset \mathcal{M}^{c}(\beta, a, \tau)$.

2.2. Assumptions and definitions. Let $f: \Omega \times[0, \tau] \times \mathbb{R}^{d} \times \mathbb{R}^{d \times n} \rightarrow \mathbb{R}^{d}$ be a function such that for all $(y, z) \in \mathbb{R}^{d} \times \mathbb{R}^{d \times n}, f(\cdot, \cdot, y, z)$ is progressively measurable, and let $\xi$ be an $\mathbb{R}^{d}$-valued $\mathscr{F}_{\tau}$-measurable random variable.

For some $\beta>0$, we assume that the triple $(\tau, \xi, f)$ satisfies the following conditions.

(H1) There exist a $\mathscr{F}_{t}$-adapted process $\theta(t)$ and a nonnegative $\mathscr{F}_{t}$-adapted process $v(t)$ such that for all $\left(y, y^{\prime}, z, z^{\prime}\right) \in \mathbb{R}^{d} \times \mathbb{R}^{d} \times \mathbb{R}^{d \times n} \times \mathbb{R}^{d \times n}$,

(i) $\left\langle y-y^{\prime}, f(t, y, z)-f\left(t, y^{\prime}, z\right)\right\rangle \leq \theta(t)\left|y-y^{\prime}\right|^{2}$,

(ii) $\left|f(t, y, z)-f\left(t, y, z^{\prime}\right)\right| \leq v(t)\left|z-z^{\prime}\right|$,

(iii) $y \mapsto f(\cdot, \cdot, y, z)$ is continuous $d t \otimes d \mathbb{P}$ a.e.

(H2) There exists $\varepsilon>0$ such that $a^{2}(t) \triangleq|\theta(t)|+v^{2}(t)>\varepsilon$.

If (H2) is not fulfilled, replace $v(t)$ by $v(t)+\sqrt{\varepsilon}$.

(H3) (i) $\xi \in L^{2}\left(\beta, a, \tau, \mathbb{R}^{d}\right)$,

(ii) $f(\cdot, 0,0) / a \in L^{2}\left(\beta, a,[0, \tau], \mathbb{R}^{d}\right)$,

(iii) $\mathbb{E}\left(e^{\beta A(\tau)}\right)<+\infty$.

(H4) There exists a positive $\mathscr{F}_{t}$-adapted process $\eta(t)$ and a positive constant $K$ such that

(i) $\eta \in L^{2}\left(\beta, a,[0, \tau], \mathbb{R}^{d}\right)$,

(ii) $|f(t, y, z)| \leq|f(t, 0, z)|+\eta(t)+K(1+|y|)$.

We end this section by specifying what we call a solution of our BSDE.

Definition 2.2. If $\tau$ is constant, then a solution of the BSDE with data $(\tau, \xi, f)$ is a pair of $\mathscr{F}_{t}$-adapted processes $\left\{\left(Y_{t}, Z_{t}\right) ; t \geq 0\right\}$ with values in $\mathbb{R}^{d} \times \mathbb{R}^{d \times n}$ such that

(J1) $(Y, Z) \in M^{c}(\beta, a, \tau)$, that is,

$$
\mathbb{E}\left(\sup _{0 \leq t \leq \tau} e^{\beta A(t)}\left|Y_{t}\right|^{2}+\int_{0}^{\tau} e^{\beta A(s)} a^{2}(s)\left|Y_{s}\right|^{2} d s+\int_{0}^{\tau} e^{\beta A(s)}\left|Z_{s}\right|^{2} d s\right)<+\infty,
$$

(J2) $Y_{t}=\xi+\int_{t}^{\tau} f\left(s, Y_{s}, Z_{s}\right) d s-\int_{t}^{\tau} Z_{s} d W_{s}$.

Definition 2.3. If $\tau$ is a random time, then a solution of the BSDE with data $(\tau, \xi, f)$ a pair of $\mathscr{F}_{t}$-adapted processes $\left\{\left(Y_{t}, Z_{t}\right), t \geq 0\right\}$, taking values in $\mathbb{R}^{d} \times \mathbb{R}^{d \times n}$ such that

(J3) $(Y, Z) \in M^{c}(\beta, a, \tau)$,

(J4) for all $T \geq t \geq 0$,

$$
Y_{t \wedge \tau}=Y_{T \wedge \tau}+\int_{t \wedge \tau}^{T \wedge \tau} f\left(s, Y_{s}, Z_{s}\right) d s-\int_{t \wedge \tau}^{T \wedge \tau} Z_{s} d W_{s},
$$

(J5) $Y_{t}=\xi$, on the set $\{t \geq \tau\}$.

\section{Existence and uniqueness on fixed time interval}

Throughout this section, $\tau$ is a fixed positive real number and $C$ will denote a positive constant which may vary from line to line. 
3.1. Uniqueness. We first state a priori estimates on the solutions.

Proposition 3.1. Under (H1), (H2), (H3), and (H4), let $(Y, Z)$ (resp., $\left.\left(Y^{\prime}, Z^{\prime}\right)\right)$ be a solution of the BSDE with data $(\tau, \xi, f)$ (resp., $\left.\left(\tau, \xi^{\prime}, f^{\prime}\right)\right)$. Put $\Delta f(t)=f\left(t, Y_{t}^{\prime}, Z_{t}^{\prime}\right)-f^{\prime}\left(t, Y_{t}^{\prime}, Z_{t}^{\prime}\right)$, $\Delta Y_{t}=Y_{t}-Y_{t}^{\prime}, \Delta Z_{t}=Z_{t}-Z_{t}^{\prime}, \Delta \xi=\xi-\xi^{\prime}$, and

$$
\Gamma=\mathbb{E}\left(e^{\beta A(\tau)}|\Delta \xi|^{2}+\frac{2}{\beta} \int_{0}^{\tau} e^{\beta A(s)} \frac{|\Delta f(s)|^{2}}{a^{2}(s)} d s\right)
$$

Then, for $\beta$ sufficiently large, the following holds:

(i) $\mathbb{E}\left(\int_{0}^{\tau} e^{\beta A(s)}\left(|\Delta f(s)|^{2} / a^{2}(s)\right) d s\right)<+\infty$,

(ii) (a) $\mathbb{E}\left(\int_{0}^{\tau} e^{\beta A(s)}\left|\Delta Z_{s}\right|^{2} d s\right) \leq 2 \Gamma$,

(b) $\mathbb{E}\left(\int_{0}^{\tau} e^{\beta A(s)} a^{2}(s)\left|\Delta Y_{s}\right|^{2} d s\right) \leq(2 /(\beta-4)) \Gamma$,

(iii) $\mathbb{E}\left(\sup _{0 \leq t \leq \tau} e^{\beta A(t)}\left|\Delta Y_{t}\right|^{2}\right) \leq C(\beta) \Gamma$,

where $C(\beta)$ is a constant which depends on $\beta$.

Proof. We assume without loss of generality that the coefficients $\theta(s)$ and $v(s)$ are the same for $f$ and $f^{\prime}$. Then, (i) follows from (H1)(ii), (H3), and (H4).

By virtue of Itô's formula, we have

$$
\begin{aligned}
e^{\beta A(t)} \mid & \left.\Delta Y_{t}\right|^{2}+\beta \int_{t}^{\tau} e^{\beta A(s)} a^{2}(s)\left|\Delta Y_{s}\right|^{2} d s+\int_{t}^{\tau} e^{\beta A(s)}\left|\Delta Z_{s}\right|^{2} d s \\
= & e^{\beta A(\tau)}|\Delta \xi|^{2}+2 \int_{t}^{\tau} e^{\beta A(s)}\left\langle\Delta Y_{s}, f\left(s, Y_{s}, Z_{s}\right)-f^{\prime}\left(s, Y_{s}^{\prime}, Z_{s}^{\prime}\right)\right\rangle d s \\
& -2 \int_{t}^{\tau} e^{\beta A(s)}\left\langle\Delta Y_{s}, \Delta Z_{s} d W_{s}\right\rangle .
\end{aligned}
$$

Therefore, (H1)(i), (H1)(ii), and Young's inequality, $2 u v \leq(\alpha / 2) u^{2}+(2 / \alpha) v^{2}$ for $\alpha>0$, lead to

$$
\begin{aligned}
2\left\langle\Delta Y_{s}\right. & \left.f\left(s, Y_{s}, Z_{s}\right)-f^{\prime}\left(s, Y_{s}^{\prime}, Z_{s}^{\prime}\right)\right\rangle \\
& \leq 2 \theta(s)\left|\Delta Y_{s}\right|^{2}+2\left|\Delta Y_{s}\right|\left[v(s)\left|\Delta Z_{s}\right|+|\Delta f(s)|\right] \\
& \leq\left(2+\frac{\beta}{2}\right) a^{2}(s)\left|\Delta Y_{s}\right|^{2}+\frac{1}{2}\left|\Delta Z_{s}\right|^{2}+\frac{2}{\beta} \frac{|\Delta f(s)|^{2}}{a^{2}(s)} .
\end{aligned}
$$

It follows that

$$
\begin{aligned}
e^{\beta A(t)} & \left|\Delta Y_{t}\right|^{2}+\left(\frac{\beta}{2}-2\right) \int_{t}^{\tau} e^{\beta A(s)} a^{2}(s)\left|\Delta Y_{s}\right|^{2} d s+\frac{1}{2} \int_{t}^{\tau} e^{\beta A(s)}\left|\Delta Z_{s}\right|^{2} d s \\
& \leq e^{\beta A(\tau)}|\Delta \xi|^{2}+\frac{2}{\beta} \int_{t}^{\tau} e^{\beta A(s)} \frac{|\Delta f(s)|^{2}}{a^{2}(s)} d s-2 \int_{t}^{\tau} e^{\beta A(s)}\left\langle\Delta Y_{s}, \Delta Z_{s} d W_{s}\right\rangle
\end{aligned}
$$


In view of (3.4), we deduce that

$$
\begin{gathered}
\left(\frac{\beta}{2}-2\right) \mathbb{E}\left(\int_{t}^{\tau} e^{\beta A(s)} a^{2}(s)\left|\Delta Y_{s}\right|^{2} d s\right)+\frac{1}{2} \mathbb{E}\left(\int_{t}^{\tau} e^{\beta A(s)}\left|\Delta Z_{s}\right|^{2} d s\right) \\
\leq \mathbb{E}\left(e^{\beta A(\tau)}|\Delta \xi|^{2}+\frac{2}{\beta} \int_{t}^{\tau} e^{\beta A(s)} \frac{|\Delta f(s)|^{2}}{a^{2}(s)} d s\right),
\end{gathered}
$$

which leads to (ii).

Now, taking $\sup _{0 \leq t \leq \tau}(\cdot)$ in (3.4), applying Burkhölder-Davis-Gundy's inequality and using (ii)(a), we obtain (iii).

Corollary 3.2. Under (H1), (H2), (H3), and (H4), the BSDEs (J1) and (J2) have at most one solution.

Proof. It is an immediate consequence of Proposition 3.1.

3.2. Existence. To reach our goal, we need first to establish the following technical result.

Proposition 3.3. Under (H1), (H2), (H3), and (H4), let $\left\{V_{t}: 0 \leq t \leq \tau\right\}$ be an $\mathscr{F}_{t^{-}}$ adapted process satisfying $\mathbb{E}\left(\int_{0}^{\tau} e^{\beta A(s)}\left|V_{s}\right|^{2} d s\right)<+\infty$. Assume moreover that there exists $\delta>0$ such that

(H5) $\mathbb{E}\left[e^{(1+\delta) \beta A(\tau)}\left(1+|\xi|^{2(1+\delta)}\right)+\left(\int_{0}^{\tau} e^{\beta A(s)} \eta^{2}(s) d s\right)^{(1+\delta)}\right]<+\infty$.

Then, there exists an $\mathscr{F}_{t}$-adapted processes $(Y, Z)$ with values in $\mathbb{R}^{d} \times \mathbb{R}^{d \times n}$ such that

(J6) $(Y, Z) \in M^{c}(\beta, a, \tau)$,

(J7) $Y_{t}=\xi+\int_{t}^{\tau} f\left(s, Y_{s}, V_{s}\right) d s-\int_{t}^{\tau} Z_{s} d W_{s}$.

Proof. In what follows, we put $h(s, y) \triangleq f\left(s, y, V_{s}\right)$ for every $s \in[0, \tau]$ and we split the proof in two parts.

Part I. We set $\bar{\xi}=e^{(\beta / 2) A(\tau)}|\xi|$ and assume that

$$
|\bar{\xi}|^{2}+\sup _{0 \leq t \leq \tau}|h(t, 0)|^{2} \leq C .
$$

Let $\varphi_{q}$ be a smooth function from $\mathbb{R}^{d}$ to $\mathbb{R}_{+}$such that $0 \leq \varphi_{q} \leq 1$ and $\varphi_{q}(x)=1$ if $|x| \leq q$, $\varphi_{q}(x)=0$ as soon as $|x| \geq q+1$. We set $q(n)=\left[\left(C+(6 \tau / \beta \varepsilon)\left(n C+n^{3}+4 K^{2} n\right)\right)^{1 / 2}\right]$, where $[r]$ is the integer part of $r$. Define

$$
h_{n}(t, y)=1_{\left\{\eta(t)+e^{\beta A(t)} \leq n\right\}} \int_{\mathbb{R}^{d}} \varphi_{q(n)+2}(y-u) h(t, y-u) \rho_{n}(u) d u,
$$

where $\rho_{n}: \mathbb{R}^{d} \rightarrow \mathbb{R}_{+}$is a sequence of smooth functions with compact support in the ball $B(0,1)$ which approximate the Dirac measure at 0 and satisfy $\int_{\mathbb{R}^{d}} \rho_{n}(u) d u=1$. Clearly, $h_{n}(t, \cdot)$ is a sequence of smooth functions with compact support satisfying the following:

(a) $h_{n}(t, \cdot)$ converges towards $h(t, \cdot)$ on compact sets,

(b) $h_{n}(t, \cdot)$ is globally stochastic Lipschitz with the coefficient $K(n, t)=a^{2}(t)+C_{n}$, where $C_{n}=(1 / 4) \alpha_{n}^{2} C+\alpha_{n}(n+K(2+q(n)+3))$ and $\alpha_{n}=\int_{\mathbb{R}^{d}}\left|\nabla \rho_{n}(u)\right| d u$, 
(c) for $|y|,\left|y^{\prime}\right| \leq q(n)+1,\left\langle y-y^{\prime}, h_{n}(t, y)-h_{n}\left(t, y^{\prime}\right)\right\rangle \leq 1_{\left\{\eta(t)+e^{\beta A(t)} \leq n\right\}} \theta(t)\left|y-y^{\prime}\right|^{2}$,

(d) $\left|h_{n}(t, y)\right| \leq 1_{\left\{\eta(t)+e^{\beta A(t)} \leq n\right\}}[|h(t, 0)|+\eta(t)+K(2+|y|)]$.

Now, set $a_{n}^{2}(t) \triangleq K(n, t)$ and $A_{n}(t) \triangleq \int_{0}^{t} a_{n}^{2}(s) d s=A(t)+C_{n} t$. One can easily check that

$$
\mathbb{E}\left(e^{\beta A_{n}(\tau)}|\xi|^{2}\right)<+\infty, \quad \mathbb{E}\left(\int_{0}^{\tau} e^{\beta A_{n}(s)} \frac{\left|h_{n}(s, 0)\right|^{2}}{a_{n}^{2}(s)} d s\right)<+\infty .
$$

Thus, in light of El Karoui and Huang [5], the equation

$$
Y_{t}^{n}=\xi+\int_{t}^{\tau} h_{n}\left(s, Y_{s}^{n}\right) d s-\int_{t}^{\tau} Z_{s}^{n} d W_{s}
$$

has a unique solution $\left(Y^{n}, Z^{n}\right)$ which belongs to the space $M^{c}\left(\beta, a_{n}, \tau\right)$. But, in view of Remark 2.1, one has $\left(Y^{n}, Z^{n}\right) \in M^{c}(\beta, a, \tau)$.

Now, we note that for all $y, 2\left\langle y, h_{n}(t, y)\right\rangle \leq(2+\beta / 2) a^{2}(t)|y|^{2}+(2 / \beta)\left(\left|h_{n}(t, 0)\right|^{2} / a^{2}(t)\right)$. Consequently, for a fixed $t \in[0, \tau]$, by applying Itô's formula to $e^{\beta[A(s)-A(t)]}\left|Y_{s}^{n}\right|^{2}$ for every $s \in[t, \tau]$, taking conditional expectation $\mathbb{E}\left(\cdot \mid \mathscr{F}_{t}\right)$ and choosing $\beta$ large enough, we obtain

$$
\left|Y_{t}^{n}\right|^{2} \leq \mathbb{E}\left(e^{\beta[A(\tau)-A(t)]}|\xi|^{2}+\frac{2}{\beta \varepsilon} \int_{t}^{\tau} e^{\beta[A(u)-A(t)]}\left|h_{n}(u, 0)\right|^{2} d u \mid \mathscr{F}_{t}\right) .
$$

But, in view of (3.6) and (d), we have

$$
\int_{t}^{\tau} e^{\beta[A(s)-A(t)]}\left|h_{n}(s, 0)\right|^{2} d s \leq\left(3 \tau C n+n^{3}+4 K^{2} n\right) .
$$

It follows that

$$
\forall t \in[0, \tau] \quad\left|Y_{t}^{n}\right|^{2} \leq C+\frac{6 \tau}{\beta \varepsilon}\left(n C+n^{3}+4 K^{2} n\right),
$$

which justifies the choice of the integer $q(n)$. The rest of this part is based on the following two lemmas.

Lemma 3.4. Under (H1), (H2), (H3), (H4), and (H5), for $\beta$ sufficiently large, the following holds:

(i) $\sup _{n \in \mathbb{N}^{*}} \mathbb{E}\left(\int_{0}^{\tau} e^{\beta A(s)} a^{2}(s)\left|Y_{s}^{n}\right|^{2} d s+\int_{0}^{\tau} e^{\beta A(s)}\left|Z_{s}^{n}\right|^{2} d s\right)<+\infty$,

(ii) $\sup _{n \in \mathbb{N}^{*}} \mathbb{E}\left(\int_{0}^{\tau} e^{\beta A(s)}\left|h_{n}\left(s, Y_{s}^{n}\right)\right|^{2} d s\right)<+\infty$,

(iii) $\sup _{n \in \mathbb{N}^{*}} \mathbb{E}\left(\sup _{0 \leq t \leq \tau} e^{(1+\delta) \beta A(t)}\left|Y_{t}^{n}\right|^{2(1+\delta)}\right)<+\infty$.

Proof. By virtue of Itô's formula, we have

$$
\begin{aligned}
e^{\beta A(t)} & \left|Y_{t}^{n}\right|^{2}+\beta \int_{t}^{\tau} e^{\beta A(s)} a^{2}(s)\left|Y_{s}^{n}\right|^{2} d s+\int_{t}^{\tau} e^{\beta A(s)}\left|Z_{s}^{n}\right|^{2} d s \\
& \leq e^{\beta A(\tau)}|\xi|^{2}+2 \int_{t}^{\tau} e^{\beta A(s)}\left|Y_{s}^{n}\right|\left|h_{n}\left(s, Y_{s}^{n}\right)\right| d s-2 \int_{t}^{\tau} e^{\beta A(s)}\left\langle Y_{s}^{n}, Z_{s}^{n} d W_{s}\right\rangle .
\end{aligned}
$$


By using Young's inequality and (d), one can check that

$$
2\left|Y_{s}^{n}\right|\left|h_{n}\left(s, Y_{s}^{n}\right)\right| \leq\left(\frac{\beta}{2}+2+\frac{K}{\varepsilon}\right) a^{2}(s)\left|Y_{s}^{n}\right|^{2}+\frac{2}{\beta} \frac{|h(s, 0)|^{2}}{a^{2}(s)}+\frac{\eta^{2}(s)+4 K^{2}}{a^{2}(s)} .
$$

If we take $\beta$ sufficiently large such that $\beta / 2-2-K / \varepsilon>0$, (3.13) becomes

$$
\begin{aligned}
e^{\beta A(t)}\left|Y_{t}^{n}\right|^{2}+\left(\frac{\beta}{2}-2-\frac{K}{\varepsilon}\right) \int_{t}^{\tau} e^{\beta A(s)} a^{2}(s)\left|Y_{s}^{n}\right|^{2} d s+\int_{t}^{\tau} e^{\beta A(s)}\left|Z_{s}^{n}\right|^{2} d s \\
\leq e^{\beta A(\tau)}|\xi|^{2}+\frac{2}{\beta} \int_{t}^{\tau} e^{\beta A(s)} \frac{|h(s, 0)|^{2}}{a^{2}(s)} d s+\frac{1}{\varepsilon} \int_{t}^{\tau} e^{\beta A(s)} \eta(s)^{2} d s \\
+\frac{4 K^{2}}{\varepsilon} \int_{t}^{\tau} e^{\beta A(s)} d s-2 \int_{t}^{\tau} e^{\beta A(s)}\left\langle Y_{s}^{n}, Z_{s}^{n} d W_{s}\right\rangle .
\end{aligned}
$$

Therefore, by using (H3), (H4), and (3.6), we derive (i).

In view of (d), (3.6), and (i), (ii) is obvious.

By taking the conditional expectation in (3.15) and using (3.6), one obtains that

$$
e^{\beta A(t)}\left|Y_{t}^{n}\right|^{2} \leq \mathbb{E}\left\{e^{\beta A(\tau)}\left(C_{1}+|\xi|^{2}\right)+\frac{1}{\varepsilon} \int_{0}^{\tau} e^{\beta A(s)} \eta(s)^{2} d s \mid \mathscr{F}_{t}\right\},
$$

where $C_{1}=2 C / \beta \varepsilon^{2}+4 K^{2} / \varepsilon^{2}$. Then, by Doob's maximal inequality (setting $p=1+\delta$ ), we derive that

$$
\mathbb{E}\left(\sup _{0 \leq t \leq \tau} e^{p \beta A(t)}\left|Y_{t}^{n}\right|^{2 p}\right) \leq C_{p} \mathbb{E}\left[e^{p \beta A(\tau)}\left(1+|\xi|^{2 p}\right)+\frac{1}{\varepsilon^{p}}\left(\int_{0}^{\tau} e^{\beta A(s)} \eta(s)^{2} d s\right)^{p}\right],
$$

which ensures (iii).

Lemma 3.5. Under (H1), (H2), (H3), (H4), and (H5), ( $\left.Y^{n}, Z^{n}\right)$ is a Cauchy sequence in $M^{c}(\beta, a, \tau)$.

Proof. Let $m \geq n$, and put $\Delta Y_{t}=Y_{t}^{m}-Y_{t}^{n}, \Delta Z_{t}=Z_{t}^{m}-Z_{t}^{n}$. By Itô's formula, we have

$$
\begin{aligned}
e^{\beta A(t)} & \left|\Delta Y_{t}\right|^{2}+\beta \int_{t}^{\tau} e^{\beta A(s)} a^{2}(s)\left|\Delta Y_{s}\right|^{2} d s+\int_{t}^{\tau} e^{\beta A(s)}\left|\Delta Z_{s}\right|^{2} d s \\
& =2 \int_{t}^{\tau} e^{\beta A(s)}\left\langle\Delta Y_{s}, h_{m}\left(s, Y_{s}^{m}\right)-h_{n}\left(s, Y_{s}^{n}\right)\right\rangle d s-2 \int_{t}^{\tau} e^{\beta A(s)}\left\langle\Delta Y_{s}, \Delta Z_{s} d W_{s}\right\rangle .
\end{aligned}
$$

We have

$$
\begin{aligned}
& 2\left\langle\Delta Y_{s}, h_{m}\left(s, Y_{s}^{m}\right)-h_{n}\left(s, Y_{s}^{n}\right)\right\rangle \\
&=2\left\langle\Delta Y_{s}, h_{m}\left(s, Y_{s}^{m}\right)-h_{m}\left(s, Y_{s}^{n}\right)\right\rangle+2\left\langle\Delta Y_{s}, h_{m}\left(s, Y_{s}^{n}\right)-h_{n}\left(s, Y_{s}^{n}\right)\right\rangle .
\end{aligned}
$$

But in view of (3.12), $\left|Y_{s}^{m}\right| \leq q(m)+1,\left|Y_{s}^{n}\right| \leq q(n)+1 \leq q(m)+1$. Therefore,

$$
\left\langle\Delta Y_{s}, h_{m}\left(s, Y_{s}^{m}\right)-h_{m}\left(s, Y_{s}^{n}\right)\right\rangle \leq 1_{\left\{\eta(s)+e^{\beta A(s)} \leq n\right\}} \theta(s)\left|\Delta Y_{s}\right|^{2} \leq 2 a^{2}(s)\left|\Delta Y_{s}\right|^{2} .
$$


It follows that

$$
\begin{aligned}
e^{\beta A(t)} & \left|\Delta Y_{t}\right|^{2}+(\beta-2) \int_{t}^{\tau} e^{\beta A(s)} a^{2}(s)\left|\Delta Y_{s}\right|^{2} d s+\int_{t}^{\tau} e^{\beta A(s)}\left|\Delta Z_{s}\right|^{2} d s \\
& \leq 2 \int_{t}^{\tau} e^{\beta A(s)}\left|\Delta Y_{s}\right|\left|h_{m}\left(s, Y_{s}^{n}\right)-h_{n}\left(s, Y_{s}^{n}\right)\right| d s-2 \int_{t}^{\tau} e^{\beta A(s)}\left\langle\Delta Y_{s}, \Delta Z_{s} d W_{s}\right\rangle .
\end{aligned}
$$

Since $\beta$ is chosen sufficiently large, we deduce that

$$
\mathbb{E}\left(\int_{0}^{\tau} e^{\beta A(s)}\left|\Delta Z_{s}\right|^{2} d s\right) \leq 2 \mathbb{E}\left(\int_{0}^{\tau} e^{\beta A(s)}\left|\Delta Y_{s}\right|\left|h_{m}\left(s, Y_{s}^{n}\right)-h_{n}\left(s, Y_{s}^{n}\right)\right| d s\right)
$$

On the other hand, Burkhölder-Davis-Gundy inequality leads to

$$
\begin{aligned}
2 \mathbb{E}\left(\sup _{0 \leq t \leq \tau} e^{\beta A(t)}\left|\int_{t}^{\tau} e^{\beta A(s)}\left\langle\Delta Y_{s}, \Delta Z_{s} d W_{s}\right\rangle\right|\right) \\
\quad \leq \frac{1}{2} \mathbb{E}\left(\sup _{0 \leq t \leq \tau} e^{\beta A(t)}\left|\Delta Y_{t}\right|^{2}\right)+2 C^{2} \mathbb{E}\left(\int_{0}^{\tau} e^{\beta A(s)}\left|\Delta Z_{s}\right|^{2} d s\right) .
\end{aligned}
$$

Therefore, taking $\sup _{0 \leq t \leq \tau}(\cdot)$ in (3.21), in view of (3.22) and (3.23), we obtain

$$
\begin{gathered}
\mathbb{E}\left(\sup _{0 \leq t \leq \tau} e^{\beta A(t)}\left|\Delta Y_{t}\right|^{2}\right)+(2 \beta-4) \mathbb{E}\left(\int_{0}^{\tau} e^{\beta A(s)} a^{2}(s)\left|\Delta Y_{s}\right|^{2} d s\right)+\mathbb{E}\left(\int_{0}^{\tau} e^{\beta A(s)}\left|\Delta Z_{s}\right|^{2} d s\right) \\
\leq C \mathbb{E}\left(\int_{0}^{\tau} e^{\beta A(s)}\left|\Delta Y_{s}\right|\left|h_{m}\left(s, Y_{s}^{n}\right)-h_{n}\left(s, Y_{s}^{n}\right)\right| d s\right) .
\end{gathered}
$$

Now, to reach our goal, it remains to show that

$$
I_{m, n}=\mathbb{E}\left(\int_{0}^{\tau} e^{\beta A(s)}\left|\Delta Y_{s}\right|\left|h_{m}\left(s, Y_{s}^{n}\right)-h_{n}\left(s, Y_{s}^{n}\right)\right| d s\right)
$$

tends to zero as $m, n \rightarrow+\infty$.

For every $M>0$, set $B_{m, n}^{M}=\left\{(s, \omega):\left|Y_{s}^{m}\right|+\left|Y_{s}^{n}\right|>M\right\}$ and $\bar{B}_{m, n}^{M}=\Omega \backslash B_{m, n}^{M}$. Let $C(\delta, \tau)$ denote a positive constant which may vary from line to line. We have

$$
I_{m, n}=I_{m, n}^{1}+I_{m, n}^{2}
$$

where

$$
\begin{aligned}
& I_{m, n}^{1}=\mathbb{E}\left(\int_{0}^{\tau} e^{\beta A(s)}\left|\Delta Y_{s}\right|\left|h_{m}\left(s, Y_{s}^{n}\right)-h_{n}\left(s, Y_{s}^{n}\right)\right| 1_{\bar{B}_{m, n}^{M}} d s\right), \\
& I_{m, n}^{2}=\mathbb{E}\left(\int_{0}^{\tau} e^{\beta A(s)}\left|\Delta Y_{s}\right|\left|h_{m}\left(s, Y_{s}^{n}\right)-h_{n}\left(s, Y_{s}^{n}\right)\right| 1_{B_{m, n}^{M}} d s\right) .
\end{aligned}
$$


We first estimate $I_{m, n}^{2}$. We use Holder's inequality and Young's inequality to obtain

$$
\begin{aligned}
I_{m, n}^{2} \leq & \frac{1}{M^{\delta}} \mathbb{E}\left\{\int_{0}^{\tau} e^{\beta A(s)}\left|\Delta Y_{s}\right|\left|h_{m}\left(s, Y_{s}^{n}\right)-h_{n}\left(s, Y_{s}^{n}\right)\right|\left(\left|Y_{s}^{m}\right|+\left|Y_{s}^{n}\right|\right)^{\delta} d s\right\} \\
\leq & \frac{1}{M^{\delta}} \mathbb{E}\left\{\int_{0}^{\tau} e^{\beta A(s)}\left(\left|Y_{s}^{m}\right|+\left|Y_{s}^{n}\right|\right)^{1+\delta}\left|h_{m}\left(s, Y_{s}^{n}\right)-h_{n}\left(s, Y_{s}^{n}\right)\right| d s\right\} \\
\leq & \frac{1}{M^{\delta}} \mathbb{E}\left\{\left(\int_{0}^{\tau} e^{\beta A(s)}\left(\left|Y_{s}^{m}\right|+\left|Y_{s}^{n}\right|\right)^{2+2 \delta} d s\right)^{1 / 2}\right. \\
& \left.\times\left(\int_{0}^{\tau} e^{\beta A(s)}\left|h_{m}\left(s, Y_{s}^{n}\right)-h_{n}\left(s, Y_{s}^{n}\right)\right|^{2} d s\right)^{1 / 2}\right\} \\
\leq & \frac{1}{2 M^{\delta}} \mathbb{E}\left\{\int_{0}^{\tau} e^{\beta A(s)}\left(\left|Y_{s}^{m}\right|+\left|Y_{s}^{n}\right|\right)^{2+2 \delta} d s+\int_{0}^{\tau} e^{\beta A(s)}\left|h_{m}\left(s, Y_{s}^{n}\right)-h_{n}\left(s, Y_{s}^{n}\right)\right|^{2} d s\right\} \\
\leq & \frac{C(\tau, \delta)}{M^{\delta}} \mathbb{E}\left\{\sup _{0 \leq t \leq \tau} e^{\beta A(t)}\left|Y_{t}^{n}\right|^{2+2 \delta}+\int_{0}^{\tau} e^{\beta A(s)}\left|h_{m}\left(s, Y_{s}^{n}\right)-h_{n}\left(s, Y_{s}^{n}\right)\right|^{2} d s\right\} \\
\leq & \frac{C(\tau, \delta)}{M^{\delta}} \sup _{n \in \mathbb{N}^{*}} \mathbb{E}\left(\sup _{0 \leq t \leq \tau} e^{\beta A(t)}\left|Y_{t}^{n}\right|^{2(1+\delta)}\right) \\
& +\frac{C(\tau, \delta)}{M^{\delta}} \sup _{n \in \mathbb{N}^{*}} \mathbb{E}\left(\int_{0}^{\tau} e^{\beta A(s)}\left|h_{m}\left(s, Y_{s}^{n}\right)-h_{n}\left(s, Y_{s}^{n}\right)\right|^{2} d s\right) .
\end{aligned}
$$

Now, in view of (d), (ii), (iii) in Lemma 3.4, we deduce that

$$
I_{m, n}^{2} \leq \frac{C(\tau, \delta)}{M^{\delta}}
$$

Since $M$ is arbitrary, $I_{m, n}^{2}$ can be made arbitrarily small by choosing $M$ large enough.

Now, we estimate $I_{m, n}^{1}$ :

$$
\begin{aligned}
I_{m, n}^{1} & \leq M \mathbb{E}\left(\int_{0}^{\tau} e^{\beta A(s)}\left|h_{m}\left(s, Y_{s}^{n}\right)-h_{n}\left(s, Y_{s}^{n}\right)\right| 1_{\left\{\left|Y_{s}^{n}\right| \leq M\right\}} d s\right) \\
& \leq M \mathbb{E}\left(\int_{0}^{\tau} \sup _{|y| \leq M} e^{\beta A(s)}\left|h_{m}(s, y)-h_{n}(s, y)\right| d s\right) .
\end{aligned}
$$

Since $h_{n}(t, \cdot)$ converges towards $h(t, \cdot)$ on compact sets and $\sup _{|y| \leq M} e^{\beta A(s)}\left|h_{n}(s, y)\right| \leq$ $\{|h(s, 0)|+\eta(s)+K(2+M)\} e^{\beta A(s)}$, Lebesgue's dominated convergence theorem ensures that the right-hand side of (3.30) tends to zero as $m, n \rightarrow+\infty$.

Hence, in view of (3.24), we conclude that

$$
\mathbb{E}\left(\sup _{0 \leq t \leq \tau} e^{\beta A(t)}\left|\Delta Y_{t}\right|^{2}\right)+(2 \beta-4) \mathbb{E}\left(\int_{0}^{\tau} e^{\beta A(s)} a^{2}(s)\left|\Delta Y_{s}\right|^{2} d s\right)+\mathbb{E}\left(\int_{0}^{\tau} e^{\beta A(s)}\left|\Delta Z_{s}\right|^{2} d s\right)
$$

tends to zero as $m, n \rightarrow+\infty$. 
Now, set $Y=\lim _{n} Y^{n}, Z=\lim _{n} Z^{n}$; we will end this part by showing that $(Y, Z)$ is a solution of the BSDE with data $(\tau, \xi, h)$. In view of the definition of the space $M^{c}(\beta, a, \tau)$, we have

$$
\begin{aligned}
& \lim _{n \rightarrow+\infty} \mathbb{E}\left(\sup _{0 \leq t \leq \tau} e^{\beta A(t)}\left|Y_{t}^{n}-Y_{t}\right|^{2}\right)=0, \\
& \lim _{n \rightarrow+\infty} \mathbb{E}\left(\int_{0}^{\tau} e^{\beta A(s)}\left|Z_{s}^{n}-Z_{s}\right|^{2} d s\right)=0 .
\end{aligned}
$$

By virtue of (3.33), we have for all $t \in[0, \tau], \int_{t}^{\tau} Z_{s}^{n} d W_{s} \rightarrow \int_{t}^{\tau} Z_{s} d W_{s}$ in $\mathbb{P}$-probability. Thus, to reach our purpose, we only need to show that

$$
\forall t \in[0, \tau], \quad \int_{t}^{\tau} h_{n}\left(s, Y_{s}^{n}\right) d s \longrightarrow \int_{t}^{\tau} h\left(s, Y_{s}\right) d s \quad \text { (in probability). }
$$

From (3.32), we deduce that there exists a subsequence $\left(Y^{n_{k}}\right)$ such that

$$
\forall t \in[0, \tau], \quad Y_{t}^{n_{k}} \longrightarrow Y_{t}, \quad \mathbb{P} \text {-a.s. }
$$

For simplicity, we assume that (3.35) holds without extracting a subsequence.

We have

$$
\begin{aligned}
& \mathbb{E}\left(\left|\int_{t}^{\tau} h_{n}\left(s, Y_{s}^{n}\right) d s-\int_{t}^{\tau} h\left(s, Y_{s}\right) d s\right|\right) \\
& \quad \leq \mathbb{E}\left(\int_{t}^{\tau}\left|h_{n}\left(s, Y_{s}^{n}\right)-h\left(s, Y_{s}^{n}\right)\right| d s\right)+\mathbb{E}\left(\int_{t}^{\tau}\left|h\left(s, Y_{s}^{n}\right)-h\left(s, Y_{s}\right)\right| d s\right) \\
& \quad=I_{1}+I_{2} .
\end{aligned}
$$

The fact that $I_{1}$ tends to zero is obtained by a similar argument as in the proof of Lemma 3.5.

Let $X_{s}^{n}=\left|h\left(s, Y_{s}^{n}\right)-h\left(s, Y_{s}\right)\right|$. We have

$$
\begin{aligned}
I_{2} & =\mathbb{E}\left(\int_{t}^{\tau} X_{s}^{n} d s\right) \\
& \leq \mathbb{E}\left(\int_{0}^{\tau} e^{\beta A(s)} X_{s}^{n} d s\right) \\
& \leq \mathbb{E}\left(\int_{0}^{\tau} e^{\beta A(s)} X_{s}^{n} 1_{\left\{X_{s}^{n} \leq r\right\}} d s\right)+\mathbb{E}\left(\int_{0}^{\tau} e^{\beta A(s)} X_{s}^{n} 1_{\left\{X_{s}^{n}>r\right\}} d s\right) .
\end{aligned}
$$

By virtue of Fubini's theorem and Chebychev's inequality, we have

$$
I_{2} \leq \int_{0}^{\tau} \mathbb{E}\left(e^{\beta A(s)} X_{s}^{n}(s) 1_{\left\{X_{s}^{n} \leq r\right\}}\right) d s+\frac{\mathbb{E}\left(\int_{0}^{\tau} e^{\beta A(s)}\left(X_{s}^{n}\right)^{2} d s\right)}{r} .
$$

Moreover, in view of (H4) and Lemma 3.4, it is clear that

$$
\sup _{n \in \mathbb{N}^{*}} \mathbb{E}\left(\int_{0}^{\tau} e^{\beta A(s)}\left(X_{s}^{n}\right)^{2} d s\right)<+\infty
$$


Therefore, the second term on the right-hand side of (3.38) can be made arbitrarily small by choosing $r$ large enough. Now, since $y \mapsto h(\cdot, y)$ is continuous, we deduce from (3.35) that for fixed $s, X_{s}^{n} \rightarrow 0$ almost surely as $n \rightarrow \infty$. So, it follows from (H2), (H3)(ii), Fubini's theorem, and Lebesgue dominated convergence theorem that the first term of (3.38) goes to zero as $n \rightarrow \infty$.

Hence, $\int_{.}^{\tau} h_{n}\left(s, Y_{s}^{n}\right) d s \rightarrow \int_{.}^{\tau} h\left(s, Y_{s}\right) d s$ (in probability), which leads to the conclusion of this part.

Part II. Let

$$
\begin{aligned}
\xi_{n} & =\frac{\inf \left(n, e^{\beta A(\tau) / 2}|\xi|\right)}{e^{\beta A(\tau) / 2}|\xi|} \xi, \\
h_{n}(t, y) & = \begin{cases}h(t, y)-h(t, 0)+\frac{\inf (n,|h(t, 0)|)}{|h(t, 0)|} h(t, 0), & \text { if } h(t, 0) \neq 0, \\
h(t, y), & \text { if } h(t, 0)=0 .\end{cases}
\end{aligned}
$$

We have $\mathbb{E}\left(e^{\beta A(\tau)}\left|\xi_{n}-\xi\right|^{2}\right) \rightarrow 0, \mathbb{E}\left(\int_{0}^{\tau} e^{\beta A(s)}\left(\left|h_{n}(s, 0)-h(s, 0)\right|^{2} / a^{2}(s)\right) d s\right) \rightarrow 0$, as $n \rightarrow+\infty$, and $\left(\xi_{n}, h_{n}\right)$ satisfies (3.6). Hence, for each $n \in \mathbb{N}^{*}$, there exists $\left(Y^{n}, Z^{n}\right)$ which satisfies (J3) and

$$
Y_{t}^{n}=\xi_{n}+\int_{t}^{\tau} h_{n}\left(s, Y_{s}^{n}\right) d s-\int_{t}^{\tau} Z_{s}^{n} d W_{s}, \quad 0 \leq t \leq \tau
$$

One can easily prove that for every $n, m \in \mathbb{N}^{*}$,

$$
\begin{aligned}
\mathbb{E}\left(\sup _{0 \leq t \leq \tau} e^{\beta A(t)}\left|Y_{t}^{n}-Y_{t}^{m}\right|^{2}+\left(\frac{\beta}{2}-2\right) \int_{0}^{\tau} e^{\beta A(s)} a^{2}(s)\left|Y_{s}^{n}-Y_{s}^{m}\right|^{2} d s\right) \\
\quad+\mathbb{E}\left(\int_{0}^{\tau} e^{\beta A(s)}\left|Z_{s}^{n}-Z_{s}^{m}\right|^{2} d s\right) \\
\leq C \mathbb{E}\left(e^{\beta A(\tau)}\left|\xi_{n}-\xi_{m}\right|^{2}+\int_{0}^{\tau} e^{\beta A(s)} \frac{\left|h_{n}(s, 0)-h_{m}(s, 0)\right|^{2}}{a^{2}(s)} d s\right) .
\end{aligned}
$$

The right-hand side tends to zero as $n, m \rightarrow+\infty$. Hence, there exists $(Y, Z)$ a pair of $\mathscr{F}_{t^{-}}$ adapted processes such that

$$
\lim _{n}\left\|\left(Y^{n}, Z^{n}\right)-(Y, Z)\right\|_{\beta, c}^{2}=0
$$

which satisfies (J3) and (J4). The proof of Proposition 3.3 is complete.

Now, we state the main result of this section.

Theorem 3.6. Under (H1), (H2), (H3), (H4), and (H5), for $\beta$ sufficiently large, the BSDEs (J1) and (J2) have a unique solution. 
Proof. For a fixed $(U, V) \in \mathcal{M}(\beta, a, \tau)$, thanks to Proposition 3.3 and Corollary 3.2, the BSDE

$$
Y_{t}=\xi+\int_{t}^{\tau} f\left(s, Y_{s}, V_{s}\right) d s-\int_{t}^{\tau} Z_{s} d W_{s}
$$

has a unique solution. So, we can define the mapping

$$
\begin{gathered}
\Pi: M(\beta, a, \tau) \longrightarrow M(\beta, a, \tau), \\
(U, V) \longmapsto \Pi(U, V)
\end{gathered}
$$

such that $\Pi(U, V)$ is the unique solution of the corresponding BSDE. Let $\left(U, V, U^{\prime}, V^{\prime}\right) \in$ $M(\beta, a, \tau) \times M(\beta, a, \tau)$ and $\Pi(U, V)=(Y, Z), \Pi\left(U^{\prime}, V^{\prime}\right)=\left(Y^{\prime}, Z^{\prime}\right)$. We combine (ii)(a) and (ii)(b) of Proposition 3.1 to obtain that

$$
\begin{gathered}
\mathbb{E}\left(\int_{0}^{\tau} e^{\beta A(s)} a^{2}(s)\left|Y_{s}-Y_{s}^{\prime}\right|^{2} d s\right)+\mathbb{E}\left(\int_{0}^{\tau} e^{\beta A(s)}\left|Z_{s}-Z_{s}^{\prime}\right|^{2} d s\right) \\
\leq\left(\frac{4}{\beta}+\frac{4}{\beta^{2}-4 \beta}\right) \mathbb{E}\left(\int_{0}^{\tau} e^{\beta A(s)}\left|V_{s}-V_{s}^{\prime}\right|^{2} d s\right) .
\end{gathered}
$$

In others words,

$$
\left\|(Y, Z)-\left(Y^{\prime}, Z^{\prime}\right)\right\|_{\beta}^{2} \leq\left(\frac{4}{\beta}+\frac{4}{\beta^{2}-4 \beta}\right)\left\|(U, V)-\left(U^{\prime}, V^{\prime}\right)\right\|_{\beta}^{2} .
$$

Hence, if $\beta$ is sufficiently large, $\Pi$ is a contracting mapping and its unique fixed point solves our BSDE.

\section{Random terminal time}

In the sequel, we assume that ( $\mathrm{H} 1)$ to $(\mathrm{H} 5)$ hold with $\tau$ being a random terminal time, which is allowed to take values in $[0,+\infty]$.

The following lemma is an important result for both the construction and the convergence of the approximation scheme.

Lemma 4.1. Let $\xi$ satisfy (H3)(i). Then,

(i) there exists $\left\{\gamma_{t}, t \geq 0\right\}$, an $L_{2}$-integrable process such that $\xi=\mathbb{E}(\xi)+\int_{0}^{\tau} \gamma(s) d W_{s}$,

(ii) the process $\left\{\xi_{t}, t \geq 0\right\}$ defined by setting $\xi_{t}=\mathbb{E}\left(\xi / \mathscr{F}_{t}\right)$ is such that $\left(\xi_{t}, \gamma_{t}\right) \in \mathcal{M}^{c}(\beta, a, \tau)$.

Proof. (i) Since $L^{2}(\beta, a, \tau) \subset L^{2}(0,0, \tau), \gamma$ is given by Itô's representation theorem.

(ii) Since $e^{(\beta / 2) A(t \wedge \tau)}\left|\xi_{t \wedge \tau}\right| \leq \mathbb{E}\left(e^{(\beta / 2) A(t \wedge \tau)}|\xi| / \mathscr{F}_{t \wedge \tau}\right)$, Doob's inequality and Jensen's inequality yield

$$
\mathbb{E}\left(\sup _{0 \leq t \leq \tau} e^{\beta A(t)}\left|\xi_{t}\right|^{2}\right) \leq 4 \mathbb{E}\left(e^{\beta A(\tau)}|\xi|^{2}\right)
$$


Now, in view of Itô's formula, Burkhölder-Davis-Gundy inequality, and Young's inequality, $2 a b \leq \alpha^{2} a^{2}+b^{2} / \alpha^{2}$, one obtains that for any $T \geq t \geq 0$,

$$
\begin{aligned}
& \beta \mathbb{E}(\left.\int_{0}^{T \wedge \tau} e^{\beta A(s)} a^{2}(s)\left|\xi_{s}\right|^{2} d s\right)+\mathbb{E}\left(\int_{0}^{T \wedge \tau} e^{\beta A(s)}\left|\gamma_{s}\right|^{2} d s\right) \\
& \quad \leq \mathbb{E}\left(e^{\beta A(T \wedge \tau)}\left|\xi_{T \wedge \tau}\right|^{2}\right)+\alpha^{2} \mathbb{E}\left(\sup _{0 \leq t \leq T \wedge \tau} e^{\beta A(t)}\left|\xi_{t}\right|^{2}\right)+\frac{C^{2}}{\alpha^{2}} \mathbb{E}\left(\int_{0}^{T \wedge \tau} e^{\beta A(s)}\left|\gamma_{s}\right|^{2} d s\right) .
\end{aligned}
$$

Then, taking $\alpha^{2}>2 C^{2}$, letting $T \rightarrow+\infty$, and using Fatou's lemma and Lebesque's dominated convergence theorem, we derive that

$$
\begin{gathered}
2 \beta \mathbb{E}\left(\int_{0}^{T \wedge \tau} e^{\beta A(s)} a^{2}(s)\left|\xi_{s}\right|^{2} d s\right)+\mathbb{E}\left(\int_{0}^{T \wedge \tau} e^{\beta A(s)}\left|\gamma_{s}\right|^{2} d s\right) \\
\leq 2 \mathbb{E}\left(e^{\beta A(\tau)}|\xi|^{2}\right)+\alpha^{2} \mathbb{E}\left(\sup _{0 \leq t \leq \tau} e^{\beta A(t)}\left|\xi_{t}\right|^{2}\right) .
\end{gathered}
$$

Therefore, (4.1) and (H3)(i) lead to (ii).

The main result of this section is the following.

Theorem 4.2. Under (H1), (H2), (H3), (H4), and (H5), the BSDEs (J3), (J4), and (J5) have a unique solution.

The existence result is based on the following sequence.

For each $n \in \mathbb{N}^{*}$, we know by Theorem 3.6 that the BSDE with data $\left(n, \xi_{n}, 1_{[0, \tau]} f\right)$ has a unique solution $\left(Y^{n}, Z^{n}\right)$ on $[0, n]$. We have

$$
Y_{t}^{n}=\xi_{n}+\int_{t \wedge \tau}^{n \wedge \tau} f\left(s, Y_{s}^{n}, Z_{s}^{n}\right) d s-\int_{t \wedge \tau}^{n \wedge \tau} Z_{s}^{n} d W_{s}, \quad \text { for } 0 \leq t \leq n
$$

We extend the sequence $\left(Y^{n}, Z^{n}\right)$ by setting $Y_{t}^{n}=\xi_{t} ; Z_{t}^{n}=\gamma_{t}$, for $t>n$.

Hence, $\left(Y^{n}, Z^{n}\right)$ solves the BSDE

$$
Y_{t}^{n}=\xi+\int_{t \wedge \tau}^{\tau} 1_{[0, n]}(s) f\left(s, Y_{s}^{n}, Z_{s}^{n}\right) d s-\int_{t \wedge \tau}^{\tau} Z_{s}^{n} d W_{s}, \quad t \geq 0
$$

We turn to convergence of the sequence $\left\{\left(Y^{n}, Z^{n}\right): n \geq 0\right\}$. To this end, we need the following lemmas.

Lemma 4.3. Put

$$
\Gamma=\mathbb{E}\left(e^{\beta A(\tau)}|\xi|^{2}+\frac{2}{\beta} \int_{0}^{\tau} e^{\beta A(s)} \frac{|f(s, 0,0)|^{2}}{a^{2}(s)} d s\right) .
$$


Then,

$$
\begin{gathered}
\sup _{n \in \mathbb{N}^{*}}\left[\frac{1}{2} \mathbb{E}\left(\int_{t \wedge \tau}^{\tau \wedge n} e^{\beta A(s)}\left|Z_{s}^{n}\right|^{2} d s\right)+\left(\frac{\beta}{2}-2\right) \int_{t \wedge \tau}^{\tau \wedge n} e^{\beta A(s)} a^{2}(s)\left|Y_{s}^{n}\right|^{2} d s\right] \leq \Gamma, \\
\sup _{n \in \mathbb{N}^{*}} \mathbb{E}\left(\sup _{0 \leq t \leq n \wedge \tau} e^{\beta A(t \wedge \tau)}\left|Y_{t}^{n}\right|^{2}\right) \leq 2 \Gamma .
\end{gathered}
$$

Proof. We apply Itô's formula to the process $e^{\beta A(t)}\left|Y_{t}^{n}\right|^{2}$ to obtain

$$
\begin{aligned}
& e^{\beta A(t \wedge \tau)}\left|Y_{t}^{n}\right|^{2}+\frac{1}{2} \int_{t \wedge \tau}^{\tau \wedge n} e^{\beta A(s)}\left|Z_{s}^{n}\right|^{2} d s+\left(\frac{\beta}{2}-2\right) \int_{t \wedge \tau}^{\tau \wedge n} e^{\beta A(s)} a^{2}(s)\left|Y_{s}^{n}\right|^{2} d s \\
& \leq e^{\beta A(n \wedge \tau)}\left|Y_{n}^{n}\right|^{2}+\frac{2}{\beta} \int_{t \wedge \tau}^{\tau \wedge n} e^{\beta A(s)} \frac{|f(s, 0,0)|^{2}}{a^{2}(s)} d s-2 \int_{t \wedge \tau}^{\tau \wedge n} e^{\beta A(s)}\left\langle Y_{s}^{n}, Z_{s}^{n} d W_{s}\right\rangle .
\end{aligned}
$$

Therefore,

$$
\begin{aligned}
& \mathbb{E}\left(e^{\beta A(t \wedge \tau)}\left|Y_{t}^{n}\right|^{2}\right)+\frac{1}{2} \mathbb{E}\left\{\int_{t \wedge \tau}^{\tau \wedge n} e^{\beta A(s)}\left|Z_{s}^{n}\right|^{2} d s+\left(\frac{\beta}{2}-2\right) \int_{t \wedge \tau}^{\tau \wedge n} e^{\beta A(s)} a^{2}(s)\left|Y_{s}^{n}\right|^{2} d s\right\} \\
& \leq \mathbb{E}\left(e^{\beta A(n \wedge \tau)}\left|Y_{n}^{n}\right|^{2}\right)+\frac{2}{\beta} \mathbb{E}\left(\int_{t \wedge \tau}^{\tau \wedge n} e^{\beta A(s)} \frac{|f(s, 0,0)|^{2}}{a^{2}(s)} d s\right) \\
& \leq \mathbb{E}\left(e^{\beta A(\tau)}|\xi|^{2}\right)+\frac{2}{\beta} \mathbb{E}\left(\int_{0}^{\tau} e^{\beta A(s)} \frac{|f(s, 0,0)|^{2}}{a^{2}(s)} d s\right),
\end{aligned}
$$

which gives (4.7). To prove (4.8), it suffices to apply Burkhölder-Davis-Gundy inequality.

Lemma 4.4. Under (H1), (H2), (H3), (H4), and (H5), $\left(Y^{n}, Z^{n}\right)_{n \in \mathbb{N}^{*}}$ is a Cauchy sequence in $\mu^{c}(\beta, a, \tau)$.

Proof. Let $(m, n) \in \mathbb{N}^{* 2}$ such that $m>n$. We put

$$
\Delta Y_{t}=Y_{t}^{m}-Y_{t}^{n} ; \quad \Delta Z_{t}=Z_{t}^{m}-Z_{t}^{n} .
$$

(i) For $n \leq t \leq m$, one has

$$
\begin{aligned}
& Y_{t}^{n}=\xi_{t}=\mathbb{E}\left(\xi \mid \mathscr{F}_{t}\right)=\xi_{m}-\int_{t \wedge \tau}^{\tau \wedge m} \gamma(s) d W_{s}, \\
& Y_{t}^{m}=\xi_{m}+\int_{t \wedge \tau}^{\tau \wedge m} f\left(s, Y_{s}^{m}, Z_{s}^{m}\right) d s-\int_{t \wedge \tau}^{\tau \wedge m} Z_{s}^{m} d W_{s} .
\end{aligned}
$$

This leads to

$$
\Delta Y_{t}=\int_{t \wedge \tau}^{\tau \wedge m} f\left(s, Y_{s}^{m}, Z_{s}^{m}\right) d s-\int_{t \wedge \tau}^{\tau \wedge m} \Delta Z_{s} d W_{s}
$$


We have

$$
\begin{aligned}
& \left|\Delta Y_{t}\right|^{2} e^{\beta A(t \wedge \tau)}+\int_{t \wedge \tau}^{\tau \wedge m} e^{\beta A(s)}\left|\Delta Z_{s}\right|^{2} d s+\beta \int_{t \wedge \tau}^{\tau \wedge m} e^{\beta A(s)}\left|\Delta Y_{s}\right|^{2} d s \\
& \quad=2 \int_{t \wedge \tau}^{\tau \wedge m} e^{\beta A(s)}\left\langle\Delta Y_{s}, f\left(s, Y_{s}^{m}, Z_{s}^{m}\right)\right\rangle d s-2 \int_{t \wedge \tau}^{\tau \wedge m} e^{\beta A(s)}\left\langle\Delta Y_{s}, \Delta Z_{s} d W_{s}\right\rangle .
\end{aligned}
$$

Since

$$
\begin{aligned}
2\left\langle\Delta Y_{s},\right. & \left.f\left(s, Y_{s}^{m}, Z_{s}^{m}\right)\right\rangle \\
= & 2\left\langle\Delta Y_{s}, f\left(s, Y_{s}^{m}, Z_{s}^{m}\right)-f\left(s, \xi_{s}, Z_{s}^{m}\right)\right\rangle \\
& +2\left\langle\Delta Y_{s}, f\left(s, \xi_{s}, Z_{s}^{m}\right)-f\left(s, \xi_{s}, \gamma_{s}\right)\right\rangle+2\left\langle\Delta Y_{s}, f\left(s, \xi_{s}, \gamma_{s}\right)\right\rangle,
\end{aligned}
$$

we get

$$
\begin{gathered}
\left|\Delta Y_{t}\right|^{2} e^{\beta A(t \wedge \tau)}+\frac{1}{2} \int_{t \wedge \tau}^{\tau \wedge m} e^{\beta A(s)}\left|\Delta Z_{s}\right|^{2} d s+\left(\frac{\beta}{2}-2\right) \int_{t \wedge \tau}^{\tau \wedge m} e^{\beta A(s)} a^{2}(s)\left|\Delta Y_{s}\right|^{2} d s \\
\leq \frac{2}{\beta} \int_{t \wedge \tau}^{\tau \wedge m} e^{\beta A(s)} \frac{\left|f\left(s, \xi_{s}, \gamma_{s}\right)\right|^{2}}{a^{2}(s)} d s-2 \int_{t \wedge \tau}^{\tau \wedge m} e^{\beta A(s)}\left\langle\Delta Y_{s}, \Delta Z_{s} d W_{s}\right\rangle .
\end{gathered}
$$

Now, in view of Burkhölder-Davis-Gundy inequality,

$$
\begin{aligned}
& \mathbb{E}\left(\sup _{n \leq t \leq m}\left|\Delta Y_{t}\right|^{2} e^{\beta A(t \wedge \tau)}\right)+\mathbb{E}\left(\int_{n \wedge \tau}^{\tau \wedge m} e^{\beta A(s)}\left|\Delta Z_{s}\right|^{2} d s\right)+C \mathbb{E}\left(\int_{n \wedge \tau}^{\tau \wedge m} e^{\beta A(s)} a^{2}(s)\left|\Delta Y_{s}\right|^{2} d s\right) \\
& \quad \leq C \mathbb{E}\left(\int_{n \wedge \tau}^{\tau} e^{\beta A(s)} \frac{\left|f\left(s, \xi_{s}, \gamma_{s}\right)\right|^{2}}{a^{2}(s)} d s\right) .
\end{aligned}
$$

By virtue of Lemma 4.1, the last term of this inequality tends to 0 as $n$ goes to infinity.

(ii) For $t \leq n \leq m$, one has

$$
\Delta Y_{t}=\Delta Y_{n}+\int_{t}^{\tau \wedge n}\left[f\left(s, Y_{s}^{m}, Z_{s}^{m}\right)-f\left(s, Y_{s}^{n}, Z_{s}^{n}\right)\right] d s-\int_{t}^{\tau \wedge n} \Delta Z_{s} d W_{s}
$$

We repeat the same calculation as in the previous case and we obtain

$$
\begin{aligned}
& \mathbb{E}\left(\sup _{0 \leq t \leq \tau \wedge n}\left|\Delta Y_{t}\right|^{2} e^{\beta A(t)}\right)+\mathbb{E}\left(\int_{0}^{\tau \wedge n} e^{\beta A(s)}\left|\Delta Z_{s}\right|^{2} d s\right)+C \mathbb{E}\left(\int_{0}^{\tau \wedge n} e^{\beta A(s)} a^{2}(s)\left|\Delta Y_{s}\right|^{2} d s\right) \\
& \quad \leq \mathbb{E}\left(\left|\Delta Y_{n}\right|^{2} e^{\beta A(n \wedge \tau)}\right) .
\end{aligned}
$$

In view of (4.17), we deduce that the right-hand side tends to zero as $n$ goes to infinity. 
Proof of Theorem 4.2

Uniqueness. Let $(Y, Z)$ and $\left(Y^{\prime}, Z^{\prime}\right)$ be two solutions of our BSDE. We set $\Delta Y_{t}=Y_{t}-Y_{t}^{\prime}$; $\Delta Z_{t}=Z_{t}-Z_{t}^{\prime}$. One has

$$
\Delta Y_{t \wedge \tau}=\Delta Y_{t \wedge T}+\int_{t \wedge \tau}^{T \wedge \tau}\left[f\left(s, Y_{s}, Z_{s}\right)-f\left(s, Y_{s}^{\prime}, Z_{s}^{\prime}\right)\right] d s-\int_{t \wedge \tau}^{T \wedge \tau} \Delta Z_{s} d W_{s}, \quad T \geq t \geq 0 .
$$

Itô's formula yields

$$
\begin{aligned}
e^{\beta A(t \wedge \tau)} & \left|\Delta Y_{t \wedge \tau}\right|^{2}+\int_{t \wedge \tau}^{T \wedge \tau} e^{\beta A(s)} a^{2}(s)\left|\Delta Y_{s}\right|^{2} d s+\int_{t \wedge \tau}^{T \wedge \tau} e^{\beta A(s)}\left|\Delta Z_{s}\right|^{2} d s \\
= & e^{\beta A(T \wedge \tau)}\left|\Delta Y_{T \wedge \tau}\right|^{2}+2 \int_{t \wedge \tau}^{T \wedge \tau} e^{\beta A(s)}\left\langle\Delta Y_{s}, f\left(s, Y_{s}, Z_{s}\right)-f\left(s, Y_{s}^{\prime}, Z_{s}^{\prime}\right)\right\rangle d s \\
& -2 \int_{t \wedge \tau}^{T \wedge \tau} e^{\beta A(s)}\left\langle\Delta Y_{s}, \Delta Z_{s} d W_{s}\right\rangle .
\end{aligned}
$$

By using condition (H1) and taking the expectation, we get for $\beta$ sufficiently large

$$
\mathbb{E}\left(e^{\beta A(t \wedge \tau)}\left|\Delta Y_{t \wedge \tau}\right|^{2}\right) \leq \mathbb{E}\left(e^{\beta A(T \wedge \tau)}\left|\Delta Y_{T \wedge \tau}\right|^{2}\right)
$$

Since $(\Delta Y, \Delta Z) \in M^{c}(\beta, a, \tau)$, we obtain by letting $T \rightarrow+\infty$ and using Lebesgue's dominated convergence theorem that

$$
\mathbb{E}\left(e^{\beta A(t \wedge \tau)}\left|\Delta Y_{t \wedge \tau}\right|^{2}\right)=0
$$

Therefore,

$$
\Delta Y_{t \wedge \tau}=0, \quad \Delta Z_{t \wedge \tau}=0
$$

Existence. We denote the limit of $\left(Y_{t}^{n}, Z_{t}^{n}\right)$ by $\left(Y_{t}, Z_{t}\right)$ and prove that $\left(Y_{t}, Z_{t}\right)$ solves the $\operatorname{BSDE}(\tau, \xi, f)$.

By virtue of Theorem 3.6, for each $T>0$, the $\operatorname{BSDE}\left(T, Y_{T}, 1_{[0, \tau]} f\right)$ has a unique solution $\left(\bar{Y}_{t}, \bar{Z}_{t}\right)$. For our purpose, it suffices to prove that $\left(Y_{t}, Z_{t}\right)=\left(\bar{Y}_{t}, \bar{Z}_{t}\right)$ for all $t \leq T$.

One has

$$
\begin{aligned}
& \bar{Y}_{t}=Y_{T}+\int_{t}^{T} 1_{[0, \tau]}(s) f\left(s, \bar{Y}_{s}, \bar{Z}_{s}\right) d s-\int_{t}^{T} \bar{Z}_{s} d W_{s}, \\
& Y_{t}^{n}=Y_{T}^{n}+\int_{t}^{T} 1_{[0, \tau]}(s) f\left(s, Y_{s}^{n}, Z_{s}^{n}\right) d s-\int_{t}^{T} Z_{s}^{n} d W_{s} .
\end{aligned}
$$

After extracting a subsequence still denoted by $Y_{t}^{n}$, it holds that

$$
\mathbb{E}\left(e^{\beta A(t \wedge \tau)}\left|\bar{Y}_{t}-Y_{t}\right|^{2}\right)=\lim _{n \rightarrow \infty} \mathbb{E}\left(e^{\beta A(t \wedge \tau)}\left|\bar{Y}_{t}-Y_{t}^{n}\right|^{2}\right)
$$


Therefore, we have to show that the right-hand side is zero. We denote $\widehat{\Delta Y}_{t}=\bar{Y}_{t}-Y_{t}^{n}$; $\widehat{\Delta Z}_{t}=\bar{Z}_{t}-Z_{t}^{n}$. One has

$$
\widehat{\Delta Y}_{t}=\widehat{\Delta Y}_{T}+\int_{t}^{T} 1_{[0, \tau]}(s)\left[f\left(s, \bar{Y}_{s}, \bar{Z}_{s}\right)-f\left(Y_{s}^{n}, Z_{s}^{n}\right)\right] d s-\int_{t}^{T} \widehat{\Delta Z}_{s} d W_{s}
$$

By using an analogous argument as in the proof of uniqueness, we have

$$
\mathbb{E}\left(e^{\beta A(t \wedge \tau)}\left|\widehat{\Delta Y}_{t}\right|^{2}\right) \leq \mathbb{E}\left(e^{\beta A(t \wedge \tau)}\left|\widehat{\Delta Y}_{T}\right|^{2}\right)
$$

Hence, $\mathbb{E}\left(e^{\beta A(t \wedge \tau)}\left|\bar{Y}_{t}-Y_{t}\right|^{2}\right) \leq \lim _{n \rightarrow \infty} \mathbb{E}\left(e^{\beta A(t \wedge \tau)}\left|Y_{T}-Y_{T}^{n}\right|^{2}\right)=0$.

Consequently, for all $t \leq T$, we have $\left(Y_{t}, Z_{t}\right)=\left(\bar{Y}_{t}, \bar{Z}_{t}\right)$.

We now state a comparison result.

Proposition 4.5 (a comparison theorem). Assume d=1. Under (H1), (H2), (H3), (H4), and $(H 5)$, let $(Y, Z)$ (resp., $\left.\left(Y^{\prime}, Z^{\prime}\right)\right)$ be a solution of the BSDE $(\tau, \xi, f)$ (resp., $\left(\tau, \xi^{\prime}, f^{\prime}\right)$ ) such that $\xi \leq \xi^{\prime}$ a.s., $f\left(t, Y_{t}^{\prime}, Z_{t}^{\prime}\right) \leq f^{\prime}\left(t, Y_{t}^{\prime}, Z_{t}^{\prime}\right) d t \times d \mathbb{P}$ a.e. Then $Y_{t} \leq Y_{t}^{\prime}$ a.s. on the set $\{t \leq \tau\}$.

Proof. We put $\Delta Y_{t}^{+}=\left(Y_{t}-Y_{t}^{\prime}\right)^{+}, \Delta Z_{t}=Z_{t}-Z_{t}^{\prime}$. Itô's formula yields

$$
\begin{aligned}
e^{\beta A(t \wedge \tau)}\left|\Delta Y_{t \wedge \tau}^{+}\right|^{2}+\int_{t \wedge \tau}^{T \wedge \tau} e^{\beta A(s)} a^{2}(s)\left|\Delta Y_{s}^{+}\right|^{2} d s+\int_{t \wedge \tau}^{T \wedge \tau} e^{\beta A(s)}\left|\Delta Z_{s}\right|^{2} d s \\
=e^{\beta A(T \wedge \tau)}\left|\Delta Y_{T \wedge \tau}\right|^{2}+2 \int_{t \wedge \tau}^{T \wedge \tau} e^{\beta A(s)}\left\langle\Delta Y_{s}^{+}, f\left(s, Y_{s}, Z_{s}\right)-f^{\prime}\left(s, Y_{s}^{\prime}, Z_{s}^{\prime}\right)\right\rangle d s \\
\quad-2 \int_{t \wedge \tau}^{T \wedge \tau} e^{\beta A(s)}\left\langle\Delta Y_{s}^{+}, \Delta Z_{s} d W_{s}\right\rangle, \quad \forall 0 \leq t \leq T, \\
\left\langle\Delta Y_{s}^{+}, f\left(s, Y_{s}, Z_{s}\right)-f^{\prime}\left(s, Y_{s}^{\prime}, Z_{s}^{\prime}\right)\right\rangle \\
\leq\left(2+\frac{\beta}{2}\right) a^{2}(s)\left|\Delta Y_{s}^{+}\right|^{2}+\frac{1}{2}\left|\Delta Z_{s}\right|^{2}+\left\langle\Delta Y_{s}^{+}, f\left(s, Y_{s}^{\prime}, Z_{s}^{\prime}\right)-f^{\prime}\left(s, Y_{s}^{\prime}, Z_{s}^{\prime}\right)\right\rangle .
\end{aligned}
$$

By analogous calculus as in the proof of uniqueness, one can prove that

$$
\mathbb{E}\left(e^{\beta A(t \wedge \tau)}\left|\Delta Y_{t \wedge \tau}^{+}\right|^{2}\right)=0,
$$

which leads to $\Delta Y_{t \wedge \tau}^{+}=0$.

Remark 4.6. When the uncertainty comes from a solution of a forward SDE, Theorem 4.2 provides a representation of the viscosity solution for elliptic PDE. More precisely, let

$$
X_{s}^{x}=x+\int_{0}^{s} b\left(X_{r}^{x}\right) d r+\int_{0}^{s} \sigma\left(X_{r}^{x}\right) d W_{r}
$$

be a diffusion process with the infinitesimal generator

$$
\mathscr{L}=\frac{1}{2}\left(\sigma \sigma^{t}\right)_{i, j}(x) \frac{\partial^{2}}{\partial x_{i} \partial x_{j}}+b_{i}(x) \frac{\partial}{\partial x_{i}} .
$$


Let $\left(Y^{x}, Z^{x}\right)$ be the unique solution of the BSDE

$$
Y_{s}^{x}=l\left(X_{\tau_{x}}^{x}\right)+\int_{s \wedge \tau_{x}}^{\tau_{x}} f\left(r, X_{r}^{x}, Y_{r}^{x}, Z_{r}^{x}\right) d r-\int_{s \wedge \tau_{x}}^{\tau_{x}} Z_{r}^{x} d W_{r}, \quad t \geq 0,
$$

where $(y, z) \mapsto f\left(\cdot, X^{x}, y, z\right)$ satisfies $(\mathrm{H} 1)-(\mathrm{H} 5)$,

$$
\sup _{x \in \bar{G}} \mathbb{E}\left(\int_{0}^{\tau_{x}} e^{\beta A(t)} \frac{\left|f\left(t, X_{t}^{x}, 0,0\right)\right|^{2}}{a^{2}(t)} d t\right)<+\infty,
$$

and $\sup _{x \in \bar{G}} \mathbb{E}\left(e^{\beta A\left(\tau_{x}\right)}\right)<+\infty$. Then, $u(x)=Y_{0}^{x}$ is continuous on $\bar{G}$ and is a viscosity solution of the semilinear system

$$
\begin{gathered}
\mathscr{L} u_{i}+f_{i}\left(x, u(x),\left(\nabla u_{i} \sigma\right)(x)\right)=0, \quad x \in G, i=1, \ldots, d, \\
u_{i}(x)=l_{i}(x), \quad x \in \partial G, i=1, \ldots, d
\end{gathered}
$$

where $G$ is an open bounded subset of $\mathbb{R}^{d}$, whose boundary $\partial G$ is of class $C^{1}, l \in C\left(\bar{G}, \mathbb{R}^{d}\right)$ and $\tau_{x}=\inf \left\{t \geq 0, X_{t}^{x} \notin \bar{G}\right\}$ finite $\mathbb{P}$-a.s.

\section{Acknowledgments}

The second author would like to thank PHYMAT of University of Toulon et du Var for its hospitality. He was supported by the Ivorian State Scholarship no. 1494 MERS/DPB/SD$\mathrm{BHCI} / \mathrm{ACA} / \mathrm{SD}$.

\section{References}

[1] K. Bahlali and É. Pardoux, Backward stochastic differential equations with locally monotone coefficient, preprint, 2001.

[2] C. Bender and M. Kohlmann, BSDE with stochastic lipschitz condition, preprint, 2000.

[3] Ph. Briand and R. Carmona, BSDEs with polynomial growth generators, J. Appl. Math. Stochastic Anal. 13 (2000), no. 3, 207-238.

[4] R. W. R. Darling and É. Pardoux, Backwards SDE with random terminal time and applications to semilinear elliptic PDE, Ann. Probab. 25 (1997), no. 3, 1135-1159.

[5] N. El Karoui and S.-J. Huang, A general result of existence and uniqueness of backward stochastic differential equations, Backward Stochastic Differential Equations (Paris, 1995-1996) (N. El Karoui and L. Mazliak, eds.), Pitman Res. Notes Math. Ser., vol. 364, Longman, Harlow, 1997, pp. 27-36.

[6] N. El Karoui, S. Peng, and M. C. Quenez, Backward stochastic differential equations in finance, Math. Finance 7 (1997), no. 1, 1-71.

[7] S. Hamadène and J. P. Lepeltier, Backward equations, stochastic control and zero-sum stochastic differential games, Stochastics Stochastics Rep. 54 (1995), no. 3-4, 221-231.

[8] É. Pardoux, Backward stochastic differential equations and viscosity solutions of systems of semilinear parabolic and elliptic PDEs of second order, Stochastic Analysis and Related Topics, VI (Geilo, 1996) (L. Decreuseufond, J. Gjerde, B. Øksendal, and A. S. Üstünel, eds.), Progr. Probab., vol. 42, Birkhäuser Boston, Massachusetts, 1998, pp. 79-127.

[9] BSDEs, weak convergence and homogenization of semilinear PDEs, Nonlinear Analysis, Differential Equations and Control (Montreal, QC, 1998) (F. H. Clarke, R. J. Stern, and G. Sabidussi, eds.), NATO Sci. Ser. C Math. Phys. Sci., vol. 528, Kluwer Academic Publishers, Dordrecht, 1999, pp. 503-549. 
[10] É. Pardoux and S. Peng, Backward stochastic differential equations and quasilinear parabolic partial differential equations, Stochastic Partial Differential Equations and Their Applications (North Carolina, 1991) (B. L. Rosovskii and R. B. Sowers, eds.), Lecture Notes in Control and Inform. Sci., vol. 176, Springer, Berlin, 1992, pp. 200-217.

K. Bahlali: UFR des Sciences, Université de Toulon-Var (UTV), BP 132, 83957 La Garde Cedex, France

E-mail address: bahlali@univ-tln.fr

A. Elouaflin: UFR de Mathématiques et Informatique, Université de Cocody, 22 BP 582 Abidjan, Côte d'Ivoire

M. N’zi: UFR de Mathématiques et Informatique, Université de Cocody, 22 BP 582 Abidjan, Côte d'Ivoire

E-mail address: nzim@ucocody.ci 


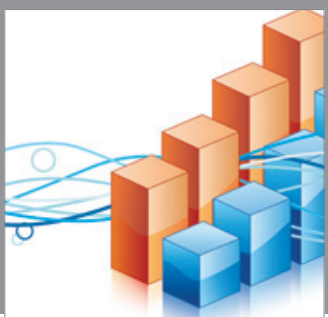

Advances in

Operations Research

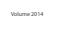

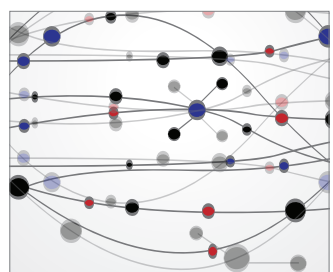

\section{The Scientific} World Journal
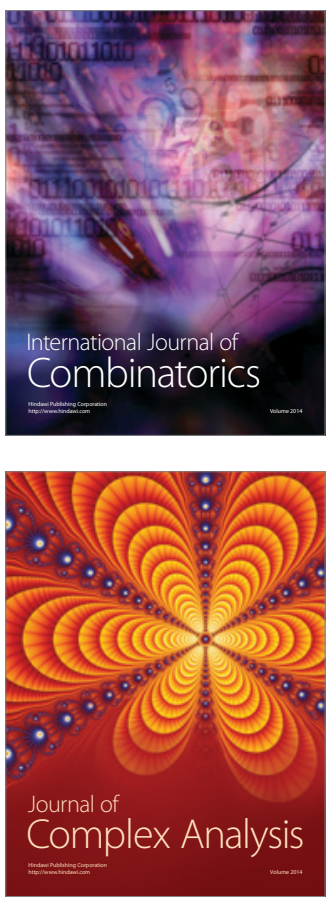

International Journal of

Mathematics and

Mathematical

Sciences
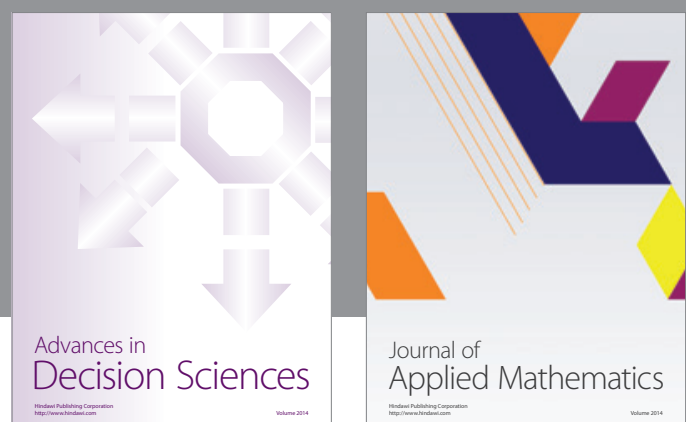

Journal of

Applied Mathematics
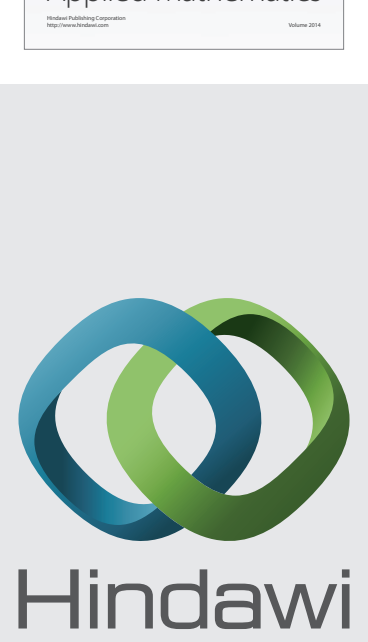

Submit your manuscripts at http://www.hindawi.com
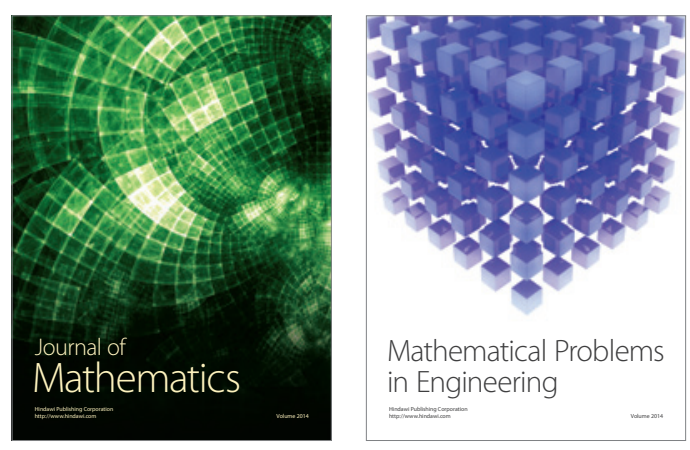

Mathematical Problems in Engineering
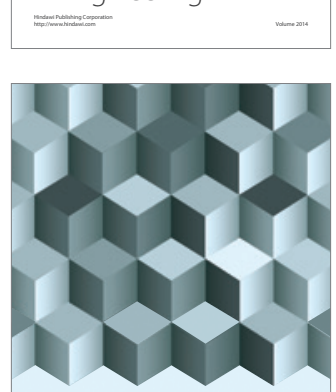

Journal of

Function Spaces
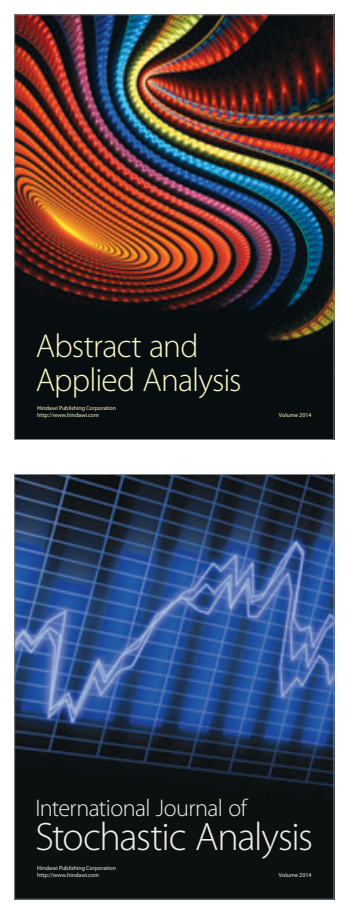

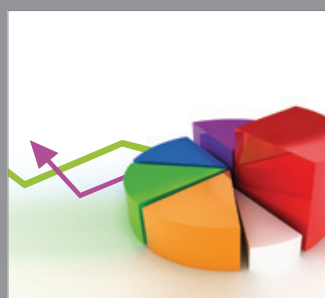

ournal of

Probability and Statistics

Promensencen
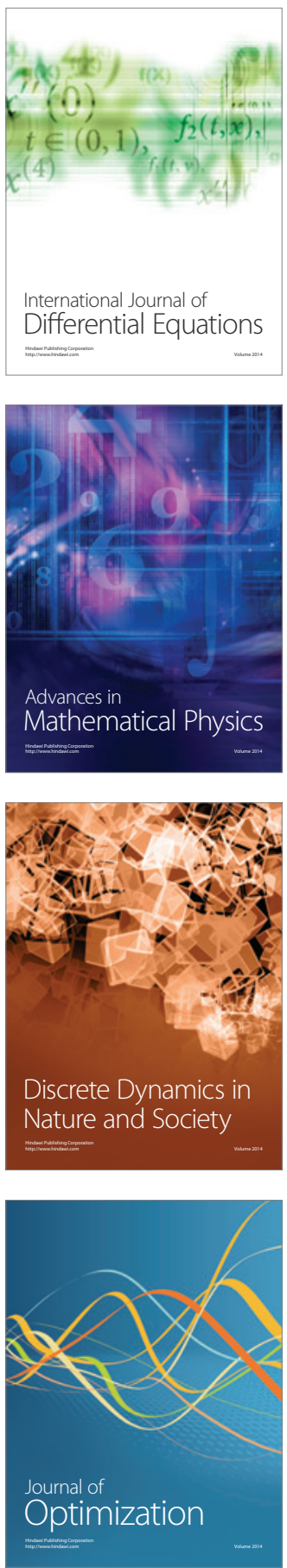